PROPOSED JOURNAL NOTE

MISMATCH STRESSES IN PRESSURE VESSELS

by Robert H. Johns

Lewis Research Center

Cleveland, Ohio

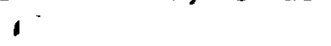

GPO PRICE

s

CFSTI PRICE(S) $\$$

Hard copy

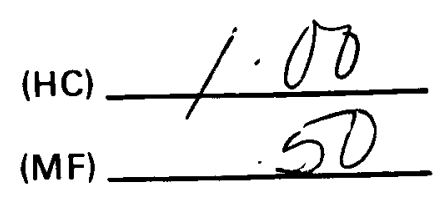

Microfiche (MF)
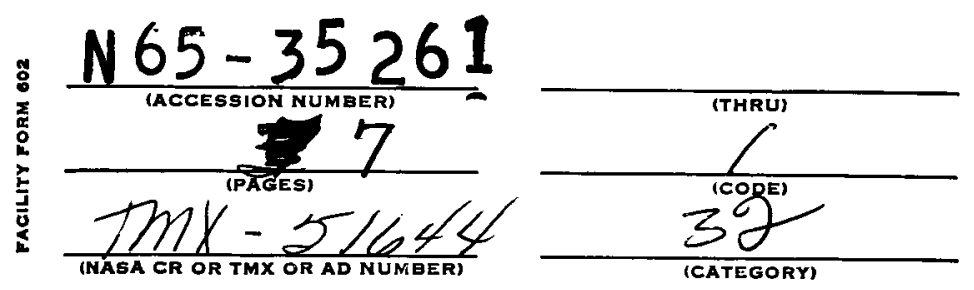

ff 653 July 65

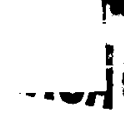

Prepared for

American Institute of Aeronautics and Astronautics Journal

April 6, 1964 


\section{MISMATCH STRESSES IN PRESSURE VESSELS}

by Robert H. Johns

Lewis Research Center

National Aeronautics and Space Administration

Cleveland, Ohio

In the manufacture of pressure vessels it is often necessary to join segments together at circumferential joints by welds or other means. Such connections may be made in lightweight flight tanks by lapping the segments and spot welding (fig. $l(a)$ ) or by butt welding the adjacent segments (fig. l(b)). The purpose of this note is to present an approximate analysis for the stresses arising at such a joint because of the mismatch or nonconcurrence of the middle surfaces of the adjoining segments that might occur. The method used is applicable to any nonshallow shell of revolution in which the meridional tangents of the two segments are parallel to each other at the junction.

The methods presented offer the following advantages: a simple closed-form solution is obtained that compares favorably with the results computed from linear edge-influence coefficients, solutions are found that are applicable to both axisymmetric and local mismatches, and an easily remembered approximate formula is suggested. The use of the equations presented herein avoids the necessity of using edge-loaded shell equations for the particular geometry being considered.

In figure $1, N_{\xi}$ is the meridional membrane force per unit length in the shells at their junction and $d$ is the distance between the midde surfaces (mismatch). The bending moment at the junction due to the eccentricity of the membrane forces is then 


$$
\begin{gathered}
-2- \\
M_{e}=N_{\xi} d
\end{gathered}
$$

If the shells are thin-walled and have equal thicknesses $h$, the eccentric moment will be distributed equally between the two shells. The maximum meridional stress occurs at the junction and is given by

$$
\sigma_{\xi, \max }=\frac{N_{\xi}}{h}+\frac{6}{h^{2}} \frac{M_{e}}{2}
$$

The maximum circumferential stress also occurs at the junction and for nonshallow shells is given by

$$
\sigma_{\theta, \max }=\frac{N_{\theta}}{h}+\frac{6 v}{h^{2}} \frac{M_{e}}{2}
$$

where $\mathbb{N}_{\theta}$ is the circumferential membrane force per unit length and $v$ is Poisson's ratio.

If the joint is a junction between two cylinders of radius a with internal pressure $p$, the foregoing equations can be written as

$$
\begin{gathered}
\mathrm{M}_{e}=\frac{\mathrm{pa}}{2} \mathrm{~d} \\
\sigma_{\xi, \max }=\frac{\mathrm{pa}}{2 \mathrm{~h}}\left(1+3 \frac{\mathrm{d}}{\mathrm{h}}\right) \\
\sigma_{\theta, \max }=\frac{\mathrm{pa}}{2 \mathrm{~h}}\left(2+3 v \frac{\mathrm{d}}{\mathrm{h}}\right)
\end{gathered}
$$

If the distortion-energy theory is used as a yiel.a. criterion, the maximum effective stress is

$$
\sigma_{e}=\sqrt{\sigma_{\xi}^{2}+\sigma_{\theta}^{2}-\sigma_{\xi} \sigma_{\theta}}
$$

Substituting equations (2a) and (3a) into (4) gives 


$$
\sigma_{e, \max }=\frac{\sqrt{3}}{2} \frac{p a}{h} \sqrt{1+3 v \frac{d}{h}+3\left(1-v+v^{2}\right)\left(\frac{d}{h}\right)^{2}}
$$

for the case of mismatch in a cylinder of constant wall thickness. From equation (4) the effective stress in the membrane region of a pressurized thin cylinder $\sigma_{e, \text { mem }}$ can be shown to be $\sqrt{3} \mathrm{pa} / 2 \mathrm{~h}$. Therefore, the quantity under the radical sign in equation (5) can be considered a stress concentration factor due to the mismatch. Dividing equation (5) by $\sqrt{3} \mathrm{pa} / 2 \mathrm{~h}=\sigma_{\mathrm{e}, \text { mem }}$ gives the stress concentration factor

$$
\operatorname{scf}=\frac{\sigma_{e, \max }}{\sigma_{e, \operatorname{mem}}}=\sqrt{1+3 v \frac{d}{h}+3\left(1-v+v^{2}\right)\left(\frac{d}{h}\right)^{2}}
$$

Equation (6) is plotted in figure 2.

The results computed from the approximate analysis presented in equation (6) agree very favorably with the results computed from the linear edge influence coefficients described in reference 1; for example, the error was less than 2 percent for 100 percent mismatch and $a / h=75$. The use of equation (6) precludes the necessity of determining edge influence coefficients and using the more rigorous equations indicated in reference 1. Contrary to the usual results obtained from edge influence coefficients the stress concentration factor is independent of the cylinder radius.

In general, the mismatch would not be uniform around the circumference; however, if it is approximately constant for several characteristic lengths $\sqrt{a h}$, the results should be sufficiently accurate for engineering purposes. If the mismatch is more of a local phenomenon, it might be more accurate to exclude the stresses due to the Poisson effect because the radial planes 
are no longer constrained to remain radial by axial symmetry, and local distortion can take place. Poisson strain due to bending is present, therefore, but not induced stress due to the Poisson effect. Consequently, for local mismatch let $v$ equal zero. Then

$$
\operatorname{scf}=\sqrt{1+3\left(\frac{a}{h}\right)^{2}}
$$

The result is identical to the one obtained from reference 2 if the overlap distance equals zero. Equation (7) is also plotted in figure 2. The radially symmetric case (eq. (6)) can be considered an upper bound and is not much greater than the case for local mismatch if the mismatch is a relatively large percentage of the shell thickness.

If the junction between the two shells is a lap joint, additional discontinuity stresses arise because the increased thickness acts as a girdle, restricting expansion due to internal pressure. These additional stresses are not accounted for in this analysis. This effect may not be serious, however, because the restriction on the radial expansion tends to reduce the hoop stress, which is generally larger than the meridional stress. Thus, the stress concentration factor is probably reduced. Also, no effect of stress concentration due to sharp corners at the mismatch is included. If the joint is butt welded, there will probably be a fillet between the two sections that minimizes this effect.

One approximation of the effect of mismatch, which is easy to remember, is that the effective stress concentration factor is 1 plus the mismatch given as a fraction of the thickness. For example, if there is 50-percent mismatch, this approximation would give a stress concentration 
factor of 1.5. For axisymmetric or relatively wide mismatch, equation (6) gives 1.43 for the stress concentration factor. For relatively local mismatch, however, equation (7) gives 1.32. In figure 2, it is apparent that this simple approximation is reasonably accurate for large percentages of mismatch but is less accurate for small mismatch.

\section{REFERENCES}

1. Johns, Robert H., and Orange, Thomas W.: Theoretical Elastic Stress Distributions Arising from Discontinuities and Edge Loads in Several Shell-Type Structures, Appendix L. INASA TR R-103, 1961.

2. Sechler, E. E.: Stress Rise Due to Offset Welds in Tension. Rep. EM 9-18, Space Tech. Labs., Inc., Aug. 1959. 


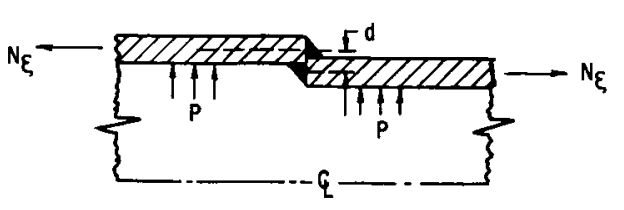

(a) Butt joint with mismatch.

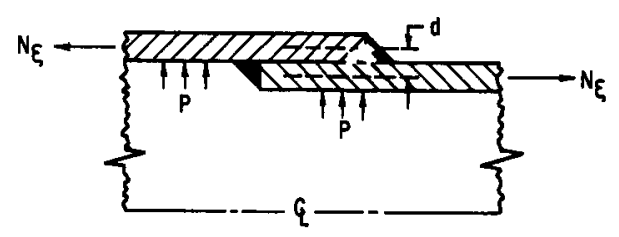

(b) Lap joint.

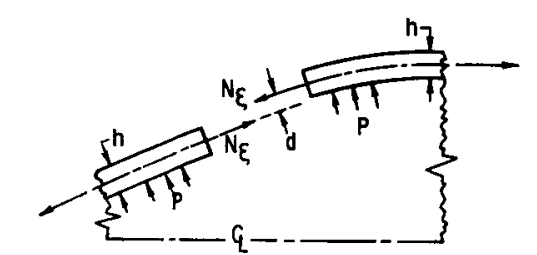

(c) Joint with mismatch in general shell of revolution.

Figure 1. - Mismatch joints and loading.

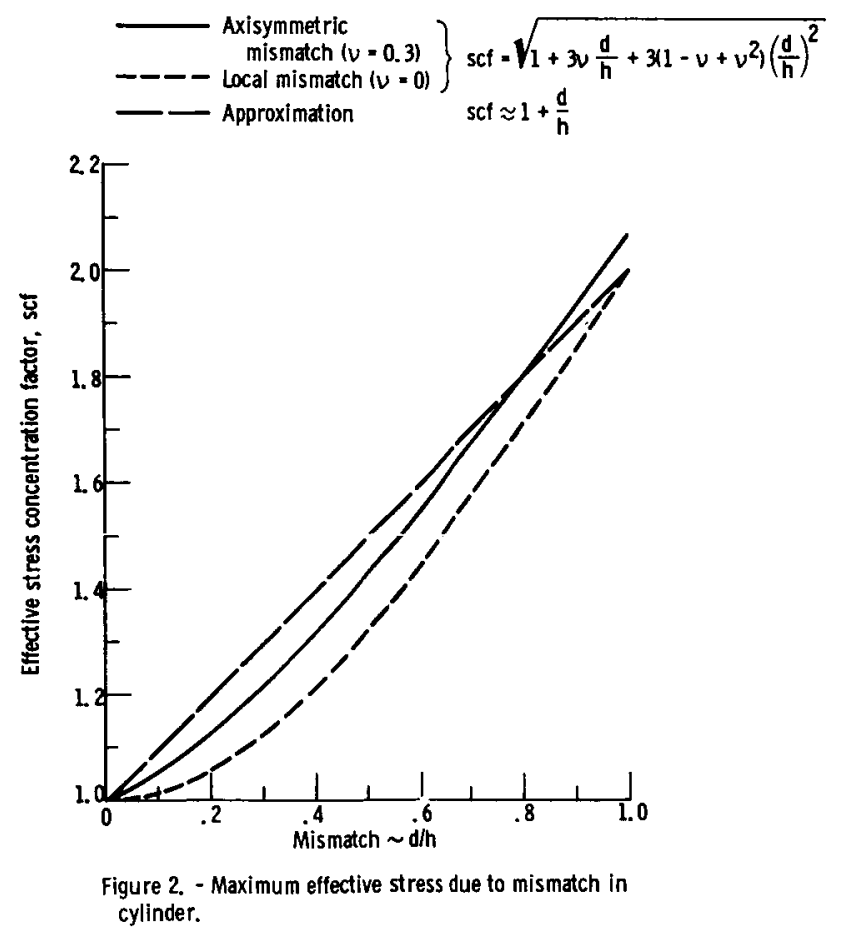

